\title{
The Mesh-Matching Algorithm : A New Automatic 3D Mesh Generator for Finite Element Analysis
}

\author{
Béatrice Couteau ${ }^{1}$, Yohan Payan ${ }^{2}$, Stéphane Lavallée ${ }^{3}$, and \\ Marie-Christine Hobatho ${ }^{1}$ \\ 1 Laboratoire de biomécanique. INSERM U305 \\ Centre Hospitalier Hôtel Dieu Toulouse - France \\ ${ }^{2}$ Laboratoire TIMC/IMAG - UMR CNRS 5525 - Faculté de Médecine \\ Domaine de la Merci, La Tronche - France \\ Yohan.Payan@imag.fr \\ 3 PRAXIM Le Grand Sablon, La Tronche - France
}

\begin{abstract}
This paper deals with Finite Element (FE) modeling of human body structures. More specifically, it focuses on the FE mesh generation process, which is a long and tedious task in the case of irregular and non-homogeneous structures. Whereas for regular and symmetrical bodies, some automatic mesh generators have been developed, no robust system is provided for living structures, which are, by definition, nonhomogeneous, irregular and patient-specific. This paper proposes a new algorithm, called the mesh-matching (M-M) algorithm, that automatically generates patient-specific 3D meshes for FE models of structures with complex geometry. It assumes that the shape which is studied is sufficiently close to a known standard model for which a mesh has been already generated by an expert. The algorithm proposes then to use a registration method, in order to infer the standard finite element mesh to the data. The M-M algorithm is tested on five human femurs.
\end{abstract}

\section{Introduction}

The rapid development of medical imaging technology has first provided the accurate visualization of the internal anatomical structures. Secondly, it has been extended to the planning and simulation of medical procedures as the navigation and immersion to 3D anatomical data-sets. Then, physical and physiological mathematical models of human organs based on medical imaging techniques were developed. The Finite Element (FE) Method is one of these modelling technique. It is a numerical analysis which provides an approximate solution to a wide variety of engineering problems, like continuum mechanics problems for example ([16]). The FE method is first based on the "meshing process" consisting in the division of the body into small volumes (called the "elements"), which are connected by "nodes". Consequently, the discretization of the problem is made by computing the mechanical equations for each node. 
In the orthopaedic domain, the clinical relevant of the Finite Element Method is the understanding of the mechanical behavior of bone structures. It may be used to analyze the influence of either a pathology or a prosthesis design on the stress field. This method has great potential for pre-clinical testing of new design implants or for simulating pre-operative implantations. But the difficulty of this method lies in the irregularities of the geometrical and mechanical properties of the bone structures. Due to those irregularities, 3D meshing is a difficult process which requires a huge amount of manual labor. For this reason, many two dimensional bone models have been developed in the orthopaedic research field, thus offering the possibility of high $2 \mathrm{D}$ mesh refinement, with a limited manual intervention $([2,3,15,21])$. In practice, bone 3D FE analysis have to respect some compromises in terms of homogeneity $([19,13])$, symmetry $([8])$ or mesh refinement $([19,20])$. Moreover, 3D models, based on average bone geometry, have also been developed $([12,22])$, loosing thus any patient-oriented specificity. An automatic method generating patient-specific 3D meshes, for FE models with irregularities, appears thus as a non trivial problem and has already been studied among the literature $([10,9]$ ). For animation purposes (facial animation for example), several algorithms have been proposed to automatically create 3 -D meshes. In the context of soft tissue modeling for surgery simulation, Cotin et al. ([4]) have used simplex-meshes representations ([6]) to mesh volumetric models. But those mesh-generation methods are not always available in standard commercial FE software as they don't include the geometrical rules of the elements. In this paper, a new method is proposed to automatically generate a 3D FE mesh of any patient anatomical structures from already defined meshes based on atlas structures. This method is called the mesh-matching (M-M) algorithm and automatically provides the 3D meshing of human structures. The method has been tested on five different human femurs. In section 1, the automatic meshgenerating method will be introduced. Section 2 will describe the procedure tested on five femurs. Finally, results will be discussed with some perspectives to quantitatively validate the method.

\section{The Mesh-Matching (M-M) Method}

Building finite element models for complex shapes is often a tedious task that requires user interaction. Our mesh-matching (M-M) algorithm assumes that the shape which is studied is sufficiently close to a known model for which a mesh has been already generated (the "standard" femur in this paper). The algorithm proposes to use a registration method to infer the finite element mesh to the data. Elastic registration is an important element of 3-D medical image analysis. In our case, the main objective of elastic registration is to deform an atlas to correspond with patient images. In a more general sense, elastic registration is the process by which an object is deformed to match another object (see [11] for more details about registration techniques). The elastic registration algorithm is defined by the estimation of a volumetric transform $\mathbf{T}$ between two coordinate systems $R_{A}$ and $R_{B}$. A point with coordinates $\left(x_{a}, y_{a}, z_{a}\right)$ in $R_{A}$ has coordinates 
$\left(x_{b}, y_{b}, z_{b}\right)=\mathbf{T}\left(x_{a}, y_{a}, z_{a}\right)$ in $R_{B}$. Obtaining such a transform is the main issue and many technical solutions exist. However, the approach always consists in minimizing the disparity between a set of features $F_{A}$ extracted in $R_{A}$ and a set of features $F_{B}$ extracted in $R_{B}$. Once such a transform has been estimated, it can be used to transform a reference object $O_{A}$ of $R_{A}$ into a new object $O_{B}$ in the coordinate system $R_{B}$ : this process is usually defined as inference. The elastic registration method we have retained for our M-M algorithm was introduced by Szeliski and Lavallée ([17]), and was characterized by three main features (see [18] for more details) :

- Representation of the elastic transform $\mathbf{T}$, with the introduction of the notion of hierarchical adaptive oct-tree splines.

- Disparity function between model and data, with the minimization of a distance between two sets of surface points.

- Optimization procedure, with the use of a combination of LevenbergMarquardt and Conjugate Gradient techniques in a hierarchical space.

Representation of the elastic transform $\mathbf{T}$ : Szeliski and Lavallée ([18]) searched a transform $\mathbf{T}$ which is the combination of a rigid-body transform $R T$, a global warping $W$ and a local displacement function $S$ built on a hierarchical and adaptive grid of displacements basis (oct-tree splines) :

$$
\mathbf{T}_{\mathbf{p}}=R T \circ W \circ S
$$

where $\mathbf{p}$ is a vector gathering the 6 parameters that define $R T$, the 12 to 30 parameters that define $W$ and the thousands of local displacement vectors that define $S$.

Disparity function between model and data : Let $\mathcal{M}=\left\{M_{i}, i=1 \ldots N_{1}\right\}$ and $\mathcal{P}=\left\{P_{i}, i=1 \ldots N_{1}\right\}$ be the sets of model and patient features, obtained by segmentation algorithms (e.g. a Canny-Deriche filtering on 3-D images). The elastic registration algorithm minimizes a least-squares criterion $E(\mathbf{p})$, as :

$$
E(\mathbf{p})=\sum_{i=1}^{N 1} \frac{1}{\sigma_{i}^{2}}\left[\operatorname{dist}\left(\mathcal{P}, \mathbf{T}_{\mathbf{p}}\left(M_{i}\right)\right)\right]^{2}+\mathcal{R}(\mathbf{p}),
$$

where $\mathcal{R}$ defines a regularization term which is applied to $S$ in order to obtain a smooth displacement function. It is, in this implementation, a weighted sum of zero order and first order regularization terms (see [18] for the exact expressions);

$\sigma_{i}^{2}$ is the variance of the noise of the measurement i ([1]);

dist is the distance between the set $\mathcal{P}$ and a point $M_{i}^{\prime}($ transformed by $\mathbf{T})$. Usually the features are simply 3-D surface points of the object surface in both the Atlas and Patient spaces. But it is also possible to use 3-D points with image gradients. In that case, the distance dist is a 6-D distance function, as proposed by Feldmar and Ayache ([7]) :

$$
\operatorname{dist}\left(\mathcal{P}, M_{i}^{\prime}\right)=\min _{P_{j} \in \mathcal{P}} d_{6 D}\left(P_{j}, M_{i}^{\prime}\right)
$$


with

$$
\begin{gathered}
d_{6 D}^{2}\left(P_{j}, M_{i}^{\prime}\right)= \\
\left(x_{P_{j}}-x_{M_{i}^{\prime}}\right)^{2}+\left(y_{P_{j}}-y_{M_{i}^{\prime}}\right)^{2}+\left(z_{P_{j}}-z_{M_{i}^{\prime}}\right)^{2}+ \\
\alpha\left(G x_{P_{j}}-G x_{M_{i}^{\prime}}\right)^{2}+\alpha\left(G y_{P_{j}}-G y_{M_{i}^{\prime}}\right)^{2}+\alpha\left(G z_{P_{j}}-G z_{M_{i}^{\prime}}\right)^{2}
\end{gathered}
$$

where $\alpha$ is a weighting factor and $P_{j}$ and $M_{i}^{\prime}$ vectors defined with $3 \mathrm{D}$ positions of coordinate and gradient points :

$$
\begin{aligned}
& P_{j}=\left(x_{P_{j}}, y_{P_{j}}, z_{P_{j}}, G x_{P_{j}}, G y_{P_{j}}, G z_{P_{j}}\right) \text { and } \\
& M_{i}^{\prime}=\left(x_{M_{i}^{\prime}}, y_{M_{i}^{\prime}}, z_{M_{i}^{\prime}}, G x_{M_{i}^{\prime}}, G y_{M_{i}^{\prime}}, G z_{M_{i}^{\prime}}\right) .
\end{aligned}
$$

Optimization procedure : The optimization of $E(\mathbf{p})$ is performed using the Levenberg-Marquardt algorithm ([14]) and a modified conjugate gradient algorithm in the hierarchical representation of $\mathbf{T}$, in order to smooth the solution and to speed up the minimization. First of all, initial registration is performed by aligning one particular point known in both data sets while rotation is simply provided by the patient coordinate systems defined in the headers of images. Therefore, rigid-body transform parameters are estimated. Then, the global warping parameters are added, the local displacement vectors for a coarse level of the octree-spline are used, and finally, the octree-spline is refined until a given resolution level is reached (see [18] for more details) .

\section{The Finite Element Modeling of Bone Structures Using the M-M Method}

Femur Acquisition Transverse CT images (Siemens, DRH2) were performed on 6 cadaveric femurs ( 2 females and 4 males). One millimeter thick slices were performed at $3 \mathrm{~mm}$ interval for the epiphyses and at $20 \mathrm{~mm}$ interval for the diaphyseal region. Each image was subjected to an edge detection to separate bone contour lines. The output file of the image processing was a neutral file (in an IGES format) containing the external contours of the cortical bone (connected by bi-parametric surfaces). The 3D surfaces of the bones were then read via the Patran Software V7.5 (MSC Nastran, Los Angeles, CA, USA).

The 3D reconstruction of one of the femur (the "standard" or "reference" femur) was manually meshed by an expert, with hexaedric ( 8 nodes) and wedge (6 nodes) finite elements. The FE model (figure 1.a) was composed of 3572 elements and has been experimentally validated by means of a vibrational technique and an extensometric measurement ([5]). The 3D surfaces of the other five femurs were automatically meshed with $2 \mathrm{D}$ elements (quads and triangles) in order to obtain the external nodes of the bone structure. Figure 1.b gives an example of this automatic meshing for one patient femur.

To allow a complete analysis of the stress field in this patient's femur, a manual volumetric meshing should be performed, which would again require a large amount of labour. Conversely, the M-M algorithm proposes to exploit the work already done on the standard femur, by inferring its $3 \mathrm{D}$ mesh to the nodes from the patient's femur. 


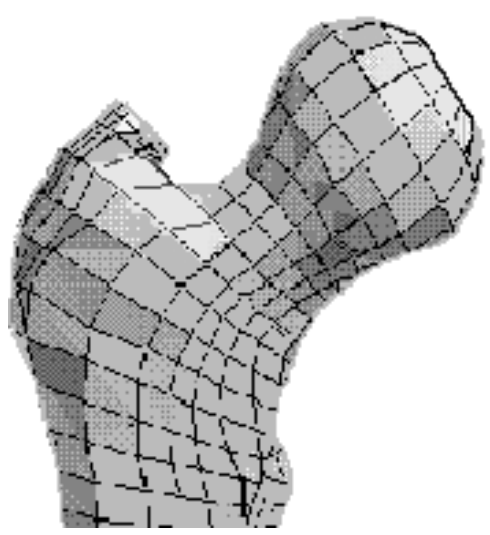

(a)

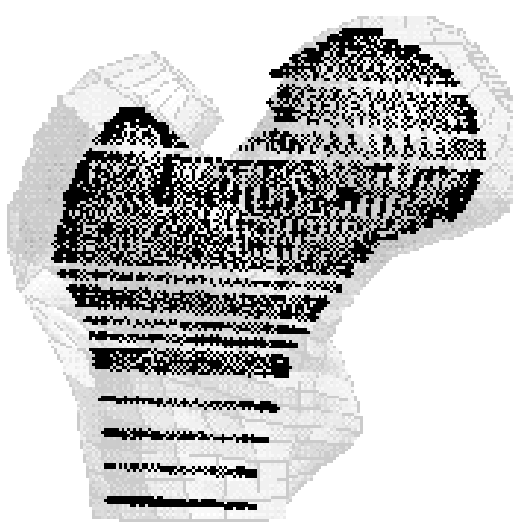

(b)

Fig. 1. 3D finite element mesh of the standard femur (a) and its superimposition with the patient's femur 3D surfacic nodes. (b)

Application of the M-M Method The 3D model of the standard human femur (figure 1.a) takes into account bone irregularities. From this 3D FE model, the points located at the surface of the bone are extracted. The matching algorithm is then required to match this standard femur surface with data collected on a new patient femur surface. Figure 2.a plots the standard femur surface (blue points) superimposed with surface points (contour lines in purple) from one of the patients femurs. The matching algorithm computes, for each patient femur, the global volumetric transformation T. Figure 2.b plots the results of one given patient, with the matching of the blue points on the femur surface.

Once surface points have been segmented on the Atlas and on the patient images, the elastic volumetric registration takes less than 30 seconds on a DEC Alpha 5000 workstation.

The last process of our M-M method is the computation of the final 3D FE meshing, of each femur. To do this, the global transformation $\mathrm{T}$ is applied to the standard 3D FE model to generate, by inference, the patient 3D meshing of the patient's femur (this step requires less than 10 seconds). Figure 3 illustrates the mesh generation of one patient based on the standard femur mesh transformation.

\section{Results and Discussion}

One of the main difficulty encountered during the mesh generation process applied to complex structures lies in the shape rules of the elements which have to respect a range of geometrical distortions. For example, FE skew angles, aspect ratio, warp angles, collapses, or twist angles have to respect some specific con- 


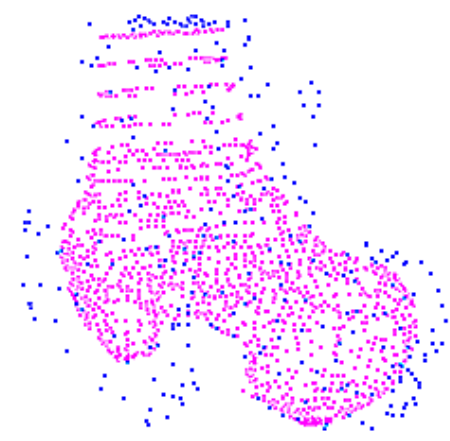

(a)

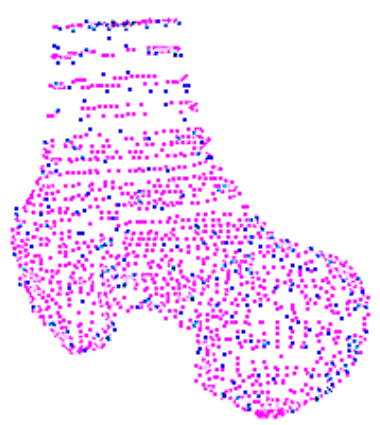

(b)

Fig. 2. Illustration of the matching of one patient's femur. (a) : Superimposition of patient's femur contour lines (purple) with points collected at the surface of the standard femur (blue). (b) : Results of the matching algorithm

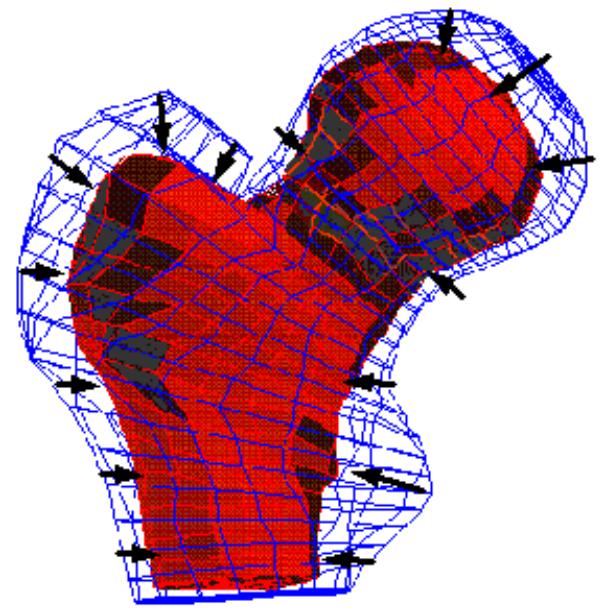

Fig. 3. Inference of the M-M algorithm : generated 3D mesh of the FE patient's femur (red), superimposed with the standard 3D mesh (blue wireframe). Black arrows illustrate the inference of the surfaces. 
straints in order to achieve a complete and correct FE analysis. The application of the M-M method on the femurs from the five patients demonstrates four successfull transformations and one failure transformation. Transformations have been tested by applying a mechanical compressive load onto the FE models of the femurs from patients. A successfull analysis means that the mesh transformation does not involve errors relative to the geometry of the elements. Conversely, a failure analysis is due to elements with a non-reasonable geometrical distortion. In our unique failed transformation, the mesh shows geometric shape failure in 13 elements, which would thus require the manipulation of an expert on those elements. Although the remaining work for the expert is simplified in comparison with building the mesh from scratch, the method is not entirely automatic for that case. It is interesting to focus here on those 13 elements which are all located at the lower part of the model. The difference between this femur and the others consists in its vertical length (15mm longer than the other ones). It would thus be interesting to quantitatively evaluate this phenomena.

\section{Conclusion}

The M-M method aims at generating automatically 3D meshes for FE analysis of human body structures. It was tested on five femurs, showing an attractive success rate. This method enables to build a FE mesh in less than 1 minute (compatible with a standard FE software). One femur among five presented geometric shape failure in 13 elements which were located at a specific part of the model. In this perspective, in order to avoid manual operation, it would become interesting to develop an algorithm that would locally modify the failed elements of the final 3D mesh. This point, associated with a more quantitative number of tests, will define the main future developments of this complete automatic mesh generator.

\section{Acknowledgements}

Richard Szeliski and Eric Bittar are acknowledged for their contributions on the elastic registration algorithm used in this paper.

\section{References}

1. P.J. Besl and N.D. McKay. A new method for registration of 3-d shapes. IEEE Transactions on Pattern Analysis and Machine Intelligence, 14(2):239-256, 1992. 1177

2. T.D. Brown, M.E. Way, F.H. Fu, and A.B. Ferguson. Finite Elements in Biomechanics. Gallagher R.H. et al. eds, New York: John Wiley, 1982. 1176

3. D.R. Carter, R. Vasu, and W.H. Harris. Stress changes in the femoral head due to porous ingrowth surface replacement arthroplasty. Journal of Biomechanics, 17:737-747, 1984. 1176 
4. S. Cotin, H. Delinguette, and N. Ayache. Real Time Volumetric Deformable Models for Surgery Simulation. Vizualisation in Biomedical Computing, K. Hohne and R. Kikinis eds., Springer, 1990. 1176

5. B. Couteau, M.C. Hobatho, R. Darmana, J.C. Brignola, and J.Y. Arlaud. Finite element modelling of the vibrational behaviour of the human femur using ct-based individualized geometrical and material properties. Journal of Biomechanics, 31:383-386, 1998. 1178

6. H. Delinguette. Simplex meshes: a general representation for $3 d$ shape reconstruction. Proceedings of Int. Conf. on Computer Vision and Pattern Recognition (CVPR'94), Seattle, USA., 1994. 1176

7. J. Feldmar and N. Ayache. Rigid, affine and locally affine registration of free-form surfaces. Int. J. of Computer Vision, 18(2):99-119, 1996. 1177

8. R. Huiskes and J.V. Heck. Stresses in the femoral head-neck region after surface replacement. a three-dimensional finite element analysis. Trans. Orthop. Res. Soc., 6:174, 1981. 1176

9. J.H. Keyak, M.G. Fourkas, J.M. Meagher, and H.B. Skinner. Validation of an automated method of three-dimensional finite element modelling of bone. J. Biomed. Eng., 15:505-509, 1993. 1176

10. J.H. Keyak, J.M. Meagher, H.B. Skinner, and C.D. Mote. Automated threedimensional finite element modelling of bone: a new method. J. Biomed. Eng., 12:389-397, 1990. 1176

11. S. Lavallée. Registration for Computer-Integrated Surgery: Methodology, State of the Art. Computer Integrated Surgery, R. Taylor, S. Lavallée, G. Burdea and R. Mosges eds., Cambridge, MA: MIT Press, 1996. 1176

12. J.C. Lotz, W.C. Hayes, and T.N. Gerhart. The structural contribution of cortical and trabecular bone in the femoral neck. Trans. Orthop. Res. Soc., 13:232, 1988. 1176

13. H. Oonishi, H. Isha, and T. Hasegawa. Mechanical analysis of the human pelvis and its application to the artificial hip joint - by means of the three dimensional finite element method. Journal of Biomechanics, 16:427-444, 1983. 1176

14. W.H. Press, B.P. Flannery, S.A. Teukolsky, and W.T. Vetterling. Numerical Recipes in C: The Art of Scientific Computing. Cambridge University Press, Cambridge, England, 1992. 1178

15. E.F. Rybicki, F.A. Simonen, and E.B. Weis. On the mathematical analysis of stress in the human femur. Journal of Biomechanics, 5:203-215, 1972. 1176

16. H.R. Schwartz. Finite Element Methods. London: Academic Press, 1984. 1175

17. R. Szeliski and S. Lavallée. Matching 3-d anatomical surfaces with non-rigid deformations using octree-splines. Geometric Methods in Computer Vision II, SPIE, San Diego., 2031:306-315, 1993. 1177

18. R. Szeliski and S. Lavallée. Matching 3-d anatomical surfaces with non-rigid deformations using octree-splines. Int. J. of Computer Vision, 18(2):171-186, 1996. 1177,1178

19. S. Valliappan, N.L Svensson, and R.D. Wood. Three dimensional stress analysis of the human femur. Comput. Biol. Med., 7:253-264, 1977. 1176

20. H.H. Vichnin and S.C. Batterman. Effects of cortical bone anisotropy on prothesis stem stresses. Trans. Orthop. Res. Soc., 7:277, 1982. 1176 
21. H. Weinans, R. Huiskes, and H. Grootenboer. The mechanical effects of fibrous tissue interposition at the cement-bone interface in tha. Trans. Orthop. Res. Soc., 13:502, 1988. 1176

22. A.M. Weinstein, J.B. Koeneman, and T.M. Hansen. Finite element analysis of a composite material hip stem. Proc. 13th Ann. Meet. Soc. Biomat., page 264, New-York, June 1987. 1176 